\title{
FACTORES DE RIESGO ASOCIADOS A FISURAS NO SINDRÓMICAS DE LABIO Y PALADAR EN NEONATOS DEL INSTITUTO NACIONAL MATERNO PERINATAL, 2012 -2017
}

\author{
Anadely Huaynate Villalobos ${ }^{1 a}$, Américo Alejandro Munayco Magallanes ${ }^{1,2, b}$
}

\begin{abstract}
RESUMEN
Objetivo. Determinar los factores de riesgo asociados a fisuras no sindrómicas de labio y paladar en neonatos del Instituto Nacional Materno Perinatal, durante el 2012 al 2017. Materiales y métodos. Estudio observacional, analítico, transversal y retrospectivo, en el que se evaluó historias clínicas de madres y neonatos, se registraron dos grupos; un grupo de casos conformado por 40 neonatos con fisuras no sindrómicas de labio y paladar y, un grupo control formado por 52 neonatos sanos. Resultados. Se encontró mayor presencia de fisuras no sindrómicas de labio y paladar en neonatos de sexo masculino (22,8\%), dándose más en el año 2016, y el diagnóstico más frecuente fue fisura del paladar duro y del paladar blando con labio leporino bilateral (Q37.4) (19,6\%). Además, se encontró que el presentar antecedentes aumenta ligeramente el riesgo de nacer con fisuras no sindrómicas de labio y paladar, y las gestantes que presentan edad materna de alto riesgo y no consumen ácido fólico tienen 1,228 y 4,891 más riesgo de desarrollar un bebé con fisuras de labio y paladar respectivamente. Conclusiones. Las fisuras de labio y paladar, se presentaron en mayor frecuencia en neonatos de sexo masculino, en el año 2016 y el diagnóstico de fisura de labio y paladar más frecuente el Q37.4, y los factores de riesgo asociados a fisuras no sindrómicas de labio y paladar fueron la edad materna y el no consumo de ácido fólico.
\end{abstract}

Palabras clave: fisuras de labio y paladar; no sindrómico; neonatos; gestantes (Fuente: DeCS BIREME).

\section{RISK FACTORS ASSOCIATED WITH NON-SYNDROMIC FISSURES OF THE LIP AND PALATE IN NEONATES OF THE NATIONAL MATERNAL PERINATAL INSTITUTE, 2012-2017}

\begin{abstract}
Objective. To determine the risk factors associated with nonsyndromic clefts of the lip and palate in neonates of the National Maternal Institute Perinatal during 2012 to 2017. Materials and methods. Analytical observational study, which evaluated the clinical histories of mothers and neonates, were recorded two groups; a group of cases consisting of 40 neonates with nonsyndromic clefts of the lip and palate, and a control group composed of 52 healthy neonates. Results. Greater presence of cleft lip and palate was found in male neonates $(22,8 \%)$ at the year 2016 and the most frequent diagnosis was cleft of the hard palate and the soft palate with liporine bilateral lip(Q37.4) (19.6\%).In addition, it was found that presenting a history slightly increases the risk of being born with nonsyndromic clefts of the lip and palate ,and pregnant women with high risk maternal age and no folic acid intake have 1,228 and 4,891 more times to develop a baby with cleft lip and palate respectively.Conclusions. Lip and palate fissures were more frequent in male neonates, with the diagnosis of lip and palate fissures being more frequent in Q37.4 located in 2016, and the risk factors associated with the nonsyndromic cleft lip and palate were maternal age and non-consumption of folicacid.
\end{abstract}

Key words: cleft lip and palate; nonsyndromic; neonates; pregnant (Source: MeSH NLM).

\section{INTRODUCCIÓN}

Las fisuras de labio y paladar son una de las malformaciones congénitas más comunes que surgen a nivel global, aproximadamente en 1 de cada 700 nacimientos con variaciones geográficas y étnicas.

Este promedio global se eleva en nuestro país ya que aquí las fisuras de labio y paladar ocurren en 1 de cada 500 nacimientos ${ }^{2}$.
Generalmente se dividen en dos grupos: fisura de paladar aislada y labio leporino con o sin fisura del paladar, grupos que afectan en gran magnitud los labios y la cavidad oral, repercutiendo notablemente en la salud ${ }^{3}$.

Es así que, la severidad de la fisura puede variar desde una muesca leve en el labio superior hasta una fisura completa que involucra las fosas nasales. Pueden ser unilaterales, afectando solo 1 lado de la cara, o bilaterales, afectando a ambos lados. También, pueden presentarse como una

Cirujano Dentista .Doctor en Odontología. Docente Facultad de Odontología-Universidad Nacional Federico Villarreal-Universidad San Martín de Porres. Odontólogo-Asistente Instituto Nacional Materno Perinatal

Citar como: Huaynate A, Munayco AA. Factores de riesgo asociados a fisuras no sindrómicas de labio y paladar en neonatos del Instituto Nacional Materno Perinatal, 2012 -2017. Rev Peru Investig Matern Perinat 2019; 8(2):9-13.

DOI: https://doi.org/10.33421/inmp.2019148 
fisura tanto en el paladar blando como en el paladar duro. La severidad de esta anomalía puede ir desde una fisura leve en la submucosa, incompetencia de la faringe, y una úvula bífida, hasta una fisura completa que involucra tanto al paladar duro como al paladar blando y las cavidades nasales ${ }^{4}$

La frecuencia de fisuras de labio y paladar también difieren según el sexo y lateralidad, hay una relación aproximada de 2:1 de hombres a mujeres para fisuras de labio y paladar, aunque levemente más mujeres que hombres tienen solo paladar hendido. Dentro de las hendiduras unilaterales de labio, la proporción de fisuras del lado izquierdo al lado derecho es también aproximadamente $2: 1^{5}$.

Clínicamente, cuando el defecto de cierre orofacial aparece con otras malformaciones dentro de patrones reconocibles se clasifica como defecto de cierre sindrómico. Si aparece como un hecho aislado o si no es posible identificar el síndrome se denomina defecto de cierre no sindrómico ${ }^{6}$.

El síndrome más común con el que puede estar relacionado es el síndrome de Pierre-Robin. Otros síndromes relacionados con fisuras de labio y paladar, son: Van der Wounde, Opitz, Stickler, Apert, DiGeorge, Charge, Waardenburg, Patau, Edwards, Meckel Treacher Collins, Goldenhar, síndrome velocardiofacial, entre otros $^{7}$.

Se establece una ocurrencia estimada de un $60 \%$ y $70 \%$ de casosdefisuras delabioypaladarcomoaisladas, sinninguna combinación con síndromes u otras malformaciones ${ }^{8}$. Estas fisuras ocurren en el periodo embrionario, en el caso de las fisuras de labio se dan a partir de la sexta semana de vida intrauterina por la no fusión de los procesos nasales mediales, y en el caso de las fisuras de paladar se dan al final de la novena semana por la no fusión de los estantes palatinos de los procesos maxilares en la línea media ${ }^{9,10}$. En el desarrollo normal, el paladar secundario se ha completado la fusión con el labio y el tabique nasal por la décima semana del desarrollo embrionario ${ }^{11}$. Al estar las fisuras de labio y paladar asociadas con niveles variables de deformidad y disfunción, implican la deficiencia ósea, de mucosa y de los músculos ${ }^{12}$,ocasionando problemas con la alimentación, el habla, la audición e integración social, disminuyendo la calidad de vida de quien la padece ${ }^{13}$.

Actualmente es posible llevar un tratamiento adecuado desde que el niño nace y puede ser corregido en diversos grados por cirugía, tratamiento dental, terapia del habla e intervención psicosocial ${ }^{8}$.

Si bien la rehabilitación es posible con buenos cuidados de calidad, las fisuras de labio y paladar dejan secuelas e inevitablemente representan una carga para el individuo, la familia y la sociedad, con sustanciales gastos en términos de salud y servicios relacionados ${ }^{11}$. En la actualidad su etiología todavía se está debatiendo.
Los avances en los estudios de investigación genética han llevado a los autores a concluir que se derivan de un origen multifactorial, resultante de la interacción de factores genéticos y ambientales ${ }^{14}$. Pese a estas cifras altas que se dan en nuestro país, no existen aquí muchos estudios con respecto a las fisuras de labio y paladar ni acerca de los factores de riesgo que predisponen a tener esta malformación.

El presente estudio pretende determinar los factores de riesgo asociados a las fisuras no sindrómicas de labio y paladar en neonatos del Instituto Nacional Materno Perinatal durante el 2012 al 2017.

\section{MATERIALES Y MÉTODOS}

Tipo de investigación. Observacional analítica de casos y controles; retrospectivo y transversal.

Población. Neonatos del Instituto Nacional Materno Perinatal de Lima nacidos durante el 2012 al 2017.

Muestra. Neonatos nacidos entre enero del 2012 a diciembre del 2017 que tuvieron el diagnóstico de fisuras no sindrómicas de labio y paladar para el grupo de casos ya que no hubo mucha población, los cuales fueron 40 . Y, para el grupo de controles se tomaron 52 neonatos sanos sin fisuras de labio y paladar nacidos entre Enero del 2012 a Diciembre del 2017.

\section{Criterios de selección}

Criterios de inclusión. Se aceptaron neonatos nacidos entre Enero del 2012 a Diciembre del 2017 con sus respectivas historias clínicas y las de sus madres, con diagnóstico de fisuras no sindrómicas de labio y paladar, para el grupo de casos y neonatos sanos, para el grupo de controles.

Criterios de exclusión. Se rechazaron neonatos nacidos de embarazos gemelares, neonatos con diagnóstico de fisuras sindrómicas de labio y paladar, y neonatos con otras malformaciones o enfermedades.

Técnica. Análisis documental.

Q35, Q36, Q37 según el CIE-10 y el número de historias clínicas de los neonatos, los cuales indican la presencia de fisuras de labio y paladar, así como la misma cantidad de historias Procedimientos. Se procedió a pedir carta de presentación a la Facultad de Odontología de la Universidad Nacional Federico Villarreal, para el Instituto Nacional Materno Perinatal (INMP). Una vez con el permiso respectivo se procedió a la recolección de datos mediante información recabada de la oficina de Estadística e informática del INMP, en donde brindaron el registro del número de las historias clínicas de madres que habían tenido neonatos nacidos del 2012 al 2017 
con diagnósticos clínicas de madres que habían tenido neonatos sanos y de sus respectivos neonatos. Con estos números, se buscó las historias clínicas en la sección de Registro del Instituto Nacional Materno Perinatal, y se anotó toda la información obtenida en una ficha de recolección de datos.

Con las fichas de recolección de datos correctamente llenadas se pudo seleccionar a los neonatos con diagnóstico de algún tipo de fisuras no sindrómicas de labio y paladar para el grupo de casos y un grupo control formado por neonatos sanos sin diagnóstico de fisuras de labio y paladar.

Procesamiento y análisis de los datos. Los datos fueron procesados en el programa Excel, donde se confeccionó una base de datos. Los resultados fueron analizados mediante el programa estadístico SPPSS versión 24,0. Se obtuvo frecuencias y porcentajes para la descripción de las variables; medidas de tendencia central y de dispersión también se calcularon y se presentaron en las tablas y gráficos. Se realizó la aplicación de la prueba de chi cuadrado y análisis de regresión logística multivariado.

\section{RESULTADOS}

En la tabla 1, se aprecia mayor número de neonatos de sexo masculino (21) en el grupo de casos, que representa el $22.8 \%$ del total. En cuanto al sexo femenino este fue menor en el grupo de casos (19) correspondientes al $20.6 \%$ del total. En el grupo control, el sexo masculino también fue más frecuente (32) correspondiente al $34.8 \%$ del total y el sexo femenino tuvo menor frecuencia (20) correspondiente al $21,6 \%$ del total.

En la tabla 2, se aprecia que los diagnósticos CIE 10 de fisuras no sindrómicas de labio y paladar más frecuentes fueron; el Q37.4 con un 19,6\%, el Q37 con un $10.9 \%$, y el Q37.9 con un 9.8\% .Y según el año, en el año 2016 el Q37.4 fue el más frecuente con un 13,0\%; el Q37 en el año 2017 (4,3\%) y, el Q37.9 en el año 2014 (5,4\%). Con respecto al grupo de controles, se encontró que durante el año 2016 hubo más frecuencia, en igual proporción al grupo de casos.

En la tabla 3, se plantea la hipótesis nula que los factores de riesgo son independientes en neonatos no sindrómico y sanos. El estadístico de contraste es menor a 0,05 para la variable antecedentes $(p=0,009)$; se rechaza la hipótesis nula y se acepta que el presentar antecedentes aumenta ligeramente el riesgo de nacer con fisuras no sindrómicas de labio y paladar (5.4\%). Para el resto de las variables no se encontró significancia estadística, lo que indica que estas variables fueron independientes de presentarse en neonatos no sindrómico y neonatos sanos.

En la tabla 4, la hipótesis nula a contrastar es que no existe asociación de los factores de riesgo y predicción de desarrollo de fisuras no sindrómicas de labio y paladar. Se encontró significancia menor a 0,05 en las variables riesgo de edad materna $(0,05)$ y, consumo de ácido fólico $(0,036)$. Es decir, las gestantes que presentan edad materna de alto riesgo y no consumo de ácido fólico tienen 1,228 y 4,891 más veces de desarrollar un producto fisuras no sindrómicas de labio y paladar respectivamente. El resumen de modelo nos permite evaluar la predicción de aparición de fisuras no sindrómicas de labio y paladar encontrándose un $41,7 \%$ de desarrollar esta patología.

Tabla 1.Frecuencia y distribución de neonatos con fisuras no sindrómicas de labio y paladar y neonatos sanos según sexo en el Instituto Nacional Materno Perinatal durante el 2012 al 2017.

\begin{tabular}{lcccc}
\hline Sexo & & $\begin{array}{c}\text { No sindrómicos } \\
\text { (casos) }\end{array}$ & $\begin{array}{c}\text { Sanos } \\
\text { (controles) }\end{array}$ & Total \\
\hline \multirow{2}{*}{ Masculino } & $\mathrm{n}$ & 21 & 32 & 53 \\
& $\%$ & $22.8 \%$ & $34,8 \%$ & $57,6 \%$ \\
& $\mathrm{n}$ & 19 & 20 & 39 \\
\multirow{2}{*}{ Femenino } & $\%$ & $20.6 \%$ & $21,6 \%$ & $42,4 \%$ \\
\multirow{2}{*}{ Total } & $\mathrm{n}$ & 40 & 52 & 92 \\
& $\%$ & $40.6 \%$ & $56,4 \%$ & $100,0 \%$ \\
\hline
\end{tabular}

Tabla 2. Frecuencia y distribución de neonatos con fisuras no sindrómicas de labio y paladar y neonatos sanos según diagnóstico y año de nacimiento en el Instituto Nacional Materno Perinatal durante el 2012 al 2017.

\begin{tabular}{|c|c|c|c|c|c|c|c|c|c|}
\hline \multirow{2}{*}{\multicolumn{3}{|c|}{ Neonatos }} & \multicolumn{6}{|c|}{ Año } & \multirow{3}{*}{$\begin{array}{c}\text { Total } \\
18 \\
(19,6 \%)\end{array}$} \\
\hline & & & \multirow{2}{*}{$\begin{array}{c}2017 \\
2 \\
(2,2 \%)\end{array}$} & \multirow{2}{*}{$\begin{array}{c}\mathbf{2 0 1 6} \\
12 \\
(13,0 \%)\end{array}$} & \multirow{2}{*}{$\begin{array}{c}2015 \\
2 \\
(2,2 \%)\end{array}$} & \multirow{2}{*}{$\begin{array}{c}\mathbf{2 0 1 4} \\
1 \\
(1,1 \%)\end{array}$} & \multirow{2}{*}{$\begin{array}{c}2013 \\
0 \\
(0 \%)\end{array}$} & \multirow{2}{*}{$\begin{array}{c}2012 \\
1 \\
(1,1 \%)\end{array}$} & \\
\hline & Q37.4 & $\mathrm{n}(\%)$ & & & & & & & \\
\hline & Q37 & $\begin{array}{c}N \\
(\%)\end{array}$ & $\begin{array}{c}4 \\
(4,3 \%)\end{array}$ & $\begin{array}{c}3 \\
(3,3 \%)\end{array}$ & $\begin{array}{c}2 \\
(2,2 \%)\end{array}$ & $\begin{array}{c}1 \\
(1,1 \%)\end{array}$ & $\begin{array}{c}0 \\
(0 \%)\end{array}$ & $\begin{array}{c}0 \\
(0 \%)\end{array}$ & $\begin{array}{c}10 \\
(10,9 \%)\end{array}$ \\
\hline \multirow[t]{3}{*}{ CASOS } & Q37.5 & $\mathrm{n}(\%)$ & $\begin{array}{c}0 \\
(0 \%)\end{array}$ & $\begin{array}{c}2 \\
(2,2 \%)\end{array}$ & $\begin{array}{l}0 \\
(0 \%)\end{array}$ & $\begin{array}{c}0 \\
(0 \%)\end{array}$ & $\begin{array}{c}0 \\
(0 \%)\end{array}$ & $\begin{array}{c}0 \\
(0 \%)\end{array}$ & $\begin{array}{c}2 \\
(2,2 \%)\end{array}$ \\
\hline & Q35.8 & n (\%) & $\begin{array}{c}0 \\
(0 \%)\end{array}$ & $\begin{array}{c}0 \\
(0 \%)\end{array}$ & $\begin{array}{c}0 \\
(0 \%)\end{array}$ & $\begin{array}{c}1 \\
(1,1 \%)\end{array}$ & $\begin{array}{c}0 \\
(0 \%)\end{array}$ & $\begin{array}{c}0 \\
(0 \%)\end{array}$ & $\begin{array}{c}1 \\
(1,1 \%)\end{array}$ \\
\hline & Q37.9 & $\mathrm{n}(\%)$ & $\begin{array}{c}0 \\
(0 \%)\end{array}$ & $\begin{array}{c}0 \\
(0 \%)\end{array}$ & $\begin{array}{c}2 \\
(2,2 \%)\end{array}$ & $\begin{array}{c}5 \\
(5,4 \%)\end{array}$ & $\begin{array}{c}2 \\
(2,2 \%)\end{array}$ & $\begin{array}{c}0 \\
(0 \%)\end{array}$ & $\begin{array}{c}9 \\
(9,8 \%)\end{array}$ \\
\hline CONTROLES & SANOS & $\mathrm{n}(\%)$ & $\begin{array}{c}12 \\
(13 \%)\end{array}$ & $\begin{array}{c}20 \\
(21,7 \%)\end{array}$ & $\begin{array}{c}7 \\
(7,6 \%)\end{array}$ & $\begin{array}{c}8 \\
(8,7 \%)\end{array}$ & $\begin{array}{c}2 \\
(2,2 \%)\end{array}$ & $\begin{array}{c}3 \\
(3,3 \%)\end{array}$ & $\begin{array}{c}52 \\
(56,5 \%)\end{array}$ \\
\hline
\end{tabular}


Tabla 3. Asociación de factores de riesgo en grupos no sindrómico y sanos.

\begin{tabular}{|c|c|c|c|c|c|c|}
\hline \multirow{2}{*}{\multicolumn{3}{|c|}{ Factores de riesgo }} & \multicolumn{2}{|l|}{ GRUPOS } & \multirow[b]{2}{*}{ Total } & \multirow[b]{2}{*}{$\mathrm{p}$-value } \\
\hline & & & No sindrómicos (casos) & $\begin{array}{c}\text { Sanos } \\
\text { (control) }\end{array}$ & & \\
\hline \multirow{2}{*}{ Edad materna } & Alto riesgo & $f(\%)$ & $9(9,8 \%)$ & $12(13 \%)$ & $21(22,8 \%)$ & \multirow{2}{*}{0,948} \\
\hline & Bajo riesgo & $f(\%)$ & $31(33,7 \%)$ & $40(43,5 \%)$ & $71(77,2 \%)$ & \\
\hline \multirow{2}{*}{ Consumo ácido fólico } & No & $f(\%)$ & $35(38 \%)$ & $41(44,6 \%)$ & $76(82,6 \%)$ & \multirow{2}{*}{0,278} \\
\hline & $\mathrm{Si}$ & $f(\%)$ & $5(5,4 \%)$ & $11(12 \%)$ & $16(17,4 \%)$ & \\
\hline \multirow{2}{*}{ Consumo de alcohol } & No & $f(\%)$ & $40(43,5 \%)$ & $52(56,5 \%)$ & $92(100 \%)$ & \\
\hline & $\mathrm{Si}$ & $f(\%)$ & - & - & - & \\
\hline \multirow{2}{*}{ Consumo de tabaco } & No & $f(\%)$ & $40(43,5 \%)$ & $52(56,5 \%)$ & $92(100 \%)$ & \multirow{2}{*}{-} \\
\hline & $\mathrm{Si}$ & $f(\%)$ & - & - & - & \\
\hline \multirow{2}{*}{ Consumo de drogas } & No & $f(\%)$ & $39(42,4 \%)$ & $52(56,5 \%)$ & $91(98,9 \%)$ & \multirow{2}{*}{0,252} \\
\hline & $\mathrm{Si}$ & $f(\%)$ & $1(1,1 \%)$ & $0(0,0 \%)$ & $1(1,1 \%)$ & \\
\hline \multirow{2}{*}{$\begin{array}{l}\text { Consumo de } \\
\text { medicamentos }\end{array}$} & Si & $f(\%)$ & $8(8,7 \%)$ & $8(8,7 \%)$ & $16(17,4 \%)$ & \multirow{2}{*}{0,424} \\
\hline & No & $f(\%)$ & $32(34,8 \%)$ & $44(47,8 \%)$ & $76(82,1 \%)$ & \\
\hline \multirow{2}{*}{ Antecedentes familiares } & No & $f(\%)$ & $35(38,0 \%)$ & $52(56,5 \%)$ & $87(94 \%)$ & \multirow{2}{*}{$0,009^{*}$} \\
\hline & $\mathrm{Si}$ & $f(\%)$ & $5(5,4 \%)$ & $0(0,0 \%)$ & $5(5,4 \%)$ & \\
\hline \multirow{2}{*}{ Controles prenatales } & $<=5$ & $f(\%)$ & $21(22,8 \%)$ & $29(31,5 \%)$ & $50(54,3 \%)$ & \multirow{2}{*}{0,755} \\
\hline & $>5$ & $f(\%)$ & $19(20,7 \%)$ & $23(25,0 \%)$ & $42(45,7 \%)$ & \\
\hline
\end{tabular}

$f=$ frecuencia absoluta

a *asociación significativa

Tabla 4. Factores de riesgo y predicción de desarrollo fisuras no sindrómicas de labio y paladar mediante regresión logística multivariable.

\begin{tabular}{|c|c|c|c|c|c|c|c|}
\hline Factores de riesgo & $\begin{array}{l}\text { Coeficiente de } \\
\text { regresión(B) }\end{array}$ & Error estándar & Wald $\left(X^{2}\right)$ & gl & Sig. & (OR) & $\begin{array}{l}\text { Resumen } \\
\text { modelo }\end{array}$ \\
\hline Edad materna & 1,480 & 816 & 3,288 & 1 & 0,050 & 1,228 & \multirow{6}{*}{$\begin{array}{c}\mathrm{R}^{2} \text { de Nagelkere } \\
41,7 \%\end{array}$} \\
\hline Controles prenatales &,- 381 &, 534 & 0,510 & 1 & 0,475 & 683 & \\
\hline Consumo de ácido fólico & 1,587 & ,757 & 4,396 & 1 & 0,036 & 4,891 & \\
\hline Consumo de drogas & 3,027 & 43993,419 & 0,000 & 1 & 1,000 & 20,643 & \\
\hline Ingesta de medicamentos & ,320 & ,393 & 0,665 & 1 & 0,415 & 1,378 & \\
\hline Antecedentes de fisuras & $-23,660$ & 17886,808 & 0,000 & 1 & 0,999 &, 000 & \\
\hline
\end{tabular}

\section{DISCUSIÓN}

El presente estudio observacional analítico, con el propósito de determinar los factores de riesgo asociados a fisuras no sindrómicas de labio y paladar en neonatos del Instituto Nacional Materno Perinatal entre el año 2012 al 2017, fue realizado en este establecimiento de salud, el cual constituye un centro de referencia a nivel nacional y sus usuarias son generalmente del centro urbano rural y marginal, lo cual permitió uniformar a la población de estudio.

Los resultados obtenidos muestran con respecto al sexo de los neonatos, mayores casos de fisuras de labio y paladar en el sexo masculino, resultado coincidente con la literatura la cual nos especifica una mayor frecuencia de fisuras de labio y paladar en los neonatos de sexo masculino ${ }^{5}$.
En la actualidad, la etiología de las fisuras no sindrómicas de labio y paladar no ha sido aclarada; y se llega a la conclusión de que estos son defectos de nacimiento multifactoriales, causados por factores genéticos y ambientales que trabajan solos o en combinación ${ }^{14}$.

La mayoría de investigaciones han evaluado el papel del ácido fólico en la aparición y la recurrencia de hendiduras orofaciales. Mientras que en algunos estudios indican que los efectos preventivos de ácido fólico, en las fisuras de labio y paladar determinan resultados aún inconsistentes ${ }^{15}$.

En el presente estudio se encontró que las gestantes que no consumieron ácido fólico, tienen mayor riesgo de que su bebé desarrolle fisuras no sindrómicas de labio y paladar hendido, coincidente con el estudio de Xu etal. ${ }^{16}$, en el que encontraron, que la cantidad apropiada de fólico ácido fue un factor de protección de hendiduras no 
sindrómicas del labio y paladar, al igual que en el estudio de Angulo et al..$^{17}$ y Dien et $-a l .{ }^{18}$ en el que se encontraron resultados similares. Discordando con el estudio de Neogi et-a/ ${ }^{19}$, en el que determinaron, que la suplementación con ácido fólico durante los primeros 3 meses de embarazo sea protector contra las fisuras de labio y paladar. Respecto a la edad materna, se encontró que las gestantes con edad de alto riesgo tienen mayor probabilidad de que su bebé desarrolle fisuras no sindrómicas de labio y paladar hendido, coincidiendo con el estudio de Xu et al ${ }^{16}$.

En cuanto a los antecedentes de fisuras de labio y paladar, se encontró que este aumenta ligeramente el riesgo de nacer con fisuras de labio y paladar no sindrómicas, similar al estudio realizado por Jamilian et $a^{20}$.

La investigación no pudo establecer asociación significativa entre fisuras no sindrómicas de labio y paladar, y el consumo de drogas, tabaco y alcohol durante el embarazo, tal vez porque las limitaciones no permitieron recabar más exhaustivamente su consumo, lo que difiere con $\mathrm{Xu}$ et $\mathrm{a}^{16}$, quien sí pudo establecer una asociación estadística significativa, ya que en su estudio se tomó como instrumento un cuestionario.

Se concluye que, en relación a la frecuencia y distribución de neonatos con fisuras no sindrómicas de labio y paladar, se encontró un mayor porcentaje en el sexo masculino con un $22.8 \%$, al igual que en el grupo de controles en el cual se encontró un $34.8 \%$.

Se evidenció, que el diagnóstico CIE 10 de fisuras no sindrómicas de labio y paladar más frecuentes fue la fisura del paladar duro y del paladar blando con labio leporino, bilateral (Q37.4) y según el año de nacimiento los diagnósticos más frecuentes fueron; el Q37.4 en el año 2016; Q37 en el año 2017 y, Q37.9 en el año 2014.

Se obtuvo asociación significativa entre los antecedentes de familiares y las fisuras no sindrómicas de labio y paladar, evidenciando que estos aumentan ligeramente el riesgo de nacer con fisuras no sindrómicas de labio y paladar.

Las gestantes que presentan edad materna de alto riesgo y no consumo de ácido fólico tienen 1,228 y 4,891 más veces de desarrollar un bebé con fisuras no sindrómicas de labio y paladar respectivamente; y los factores de riesgo asociados a fisuras no sindrómicas de labio y paladar en neonatos del Instituto Nacional Materno Perinatal durante el 2012 al 2017, fueron el no consumo de ácido fólico y la edad materna.

Financiamiento: Autofinanciado.

Conflicto de interés: Los autores declaran no tener algún conflicto de intereses.

\section{REFERENCIAS BIBLIOGRÁFICAS}

1. Mossey PA y Modell B. Epidemiology of Oral Clefts 2012: An International Perspective. Front Oral Biol. 2012; 16(1), 1-18. Navarro CE. CIRPLAST: Cleft Lip and Palate Missions in Peru. J Craniofac Surg. 2015; 26(4):1109-1111. Buyu Y, Manyama M, Chandika A y Gilyoma J. Orofacial clefts at Bugando Medical Centre: associated factors and postsurgical complications. Cleft Palate Craniofac J.2012; 49(6): 736-740.

2. Merritt L. Part 1. Understanding the embryology and genetics of cleft lip and palate. Adv Neonatal Care. 2005; 5(2): 64-71. Marazita ML y Mooney MP. Current concepts in the embryology and genetics of cleft lip and cleft palate. Clin Plast Surg. 2004; 31(2):125-140.

3. Díaz GH y Díaz GJ. Defectos de cierre orofaciales: paladar hendido y labio leporino. Semergen. 2013; 39(5): 267-271.

4. Venkatesh R. Syndromes and anomalies associated with cleft. Indian J Plast Surg. 2009; 42(3): 51-55. Dixon MJ, Marazita ML, Beaty TH y Murray JC. Cleft lip and palate: understanding genetic and environmental influences. Nat Rev Genet. 2011; 12(3):167-78.

5. Sperber GH. Formation of the primary and secondary palate. En Wyszynski DF, editor. Cleft lip and palate. From origin to treatment. Estados Unidos: Oxford University Press; 2002. p. 5-24. Kallén B. Epidemiology of human congenital malformations. Suiza: Spinger; 2014.

6. Mossey PA, Little J, Munger RG, Dixon MJ y Shaw WC. Cleft lip and palate. Lancet. 2009; 374(9703):1773-1785.

7. Shkoukan MA., Lawrence LA, Liebertz DJ y Svider PF. Cleft palate: A clinical review. Birth Defects Res. 2014; 102:333342.

8. Valero L. Aspectos psicológicos de la persona con fisura palatina.Rev. Logop. Foniatr. Audiol. 2002; 22 (1): 2-8.

9. Francois-Fiquet C, Poli-Merol ML, Nguyen P, Landais E, Gaillard D y Doco-Fenzy M. Role of angiogenesis-related genes in cleft lip/palate: Review of the literature. Int J Pediatr Otorhinolaryngol. 2014; 78(10):1579-1585.

10. Wehby GL y Murray JC. Folic acid and orofacial clefts: a review of the evidence. Oral Dis. 2010; 16(1):11-19.

11. Xu DP, Qu WD, Sun C, Cao RY, Liu DW y Du PG. A Study on Environmental Factors for Nonsyndromic Cleft Lip and/or Palate. J Craniofac Surg. 2017; 29(2):364-367.

12. Angulo E, Acosta L, Guadrón A, Cañizales A, González F, Osuna I y Murillo J. Maternal risk factors associated with the development of cleft lip and cleft palate in Mexico: a casecontrol study. Iran J Otorhinolaryngol. 2017; 29(4):189-195.

13. Dien VH, McKinney CM, Pisek A y Pitiphat W. Maternal exposures and risk of oral clefts in South Vietnam. Birth Defects Res.2017; 110(6):527-537.

14. Neogi SB, Singh S, Pallepogula DR, Pant $H$, Kolli SR, Bharti $P$, et al. Factors for Orofacial Clefts in India: A Case-Control Study. Birth Defects Res. 2017; 109(16):1284-1291.

15. Jamilian A, Sarkarat F, Jafari M, Neshandar M, Amini E, Khosravi S y Ghassemi A. Family history and risk factors for cleft lip and palate patients and their associated anomalies. Stomatologija. 2017; 19(3):78-83.

Correspondencia: Américo Alejandro Munayco Magallanes Dirección: Av. Paso de los Andes N ${ }^{\circ} 1191$. Pueblo Libre. Perú. Correo electrónico: a_munayco@hotmail.com Teléfono: 261-0583 